\title{
Quetiapine associated dyslipidemia and hyperglycemia in a pediatric overweight patient
}

\author{
Elizabeth Carreño, Vivekananda Dasari, Aditya Badheka, \\ Shanti Yogananda, Swati Dave-Sharma
}

\begin{abstract}
Introduction: Atypical antipsychotics like quetiapine, can cause undesirable alterations in lipid profile and hyperglycemia. They are associated with metabolic and cardiovascularrelated adverse events in pediatric population, especially when multiple antipsychotics or classes of psychotropic medications are coprescribed. Case Report: A 15-year-old, overweight, Hispanic male child was referred to the endocrinology clinic by his primary care provider due to high serum cholesterol, triglyceride and glucose levels. Past history was significant for autism, anxiety with depression and attention deficit disorder for which he was getting psychiatric care and was receiving quetiapine $50 \mathrm{mg}$ daily for more than two years. Physical examination was significant for increased adiposity and overweight. Fasting laboratory studies showed elevated cholesterol and triglycerides. In addition to lifestyle modification including diet and exercise, quetiapine was discontinued and replaced by aripiprazole. Repeat testing after four months showed normalized cholesterol and triglyceride levels. Conclusion: The use of atypical antipsychotics prescribed to children and adolescents with neuropsychiatric disorders has been associated with various adverse effects, including significant weight gain. These adverse
\end{abstract}

Elizabeth Carreño ${ }^{1}$, Vivekananda Dasari ${ }^{1}$, Aditya Badheka $^{1}$, Shanti Yogananda ${ }^{1}$, Swati Dave-Sharma ${ }^{1}$

Affiliations: ${ }^{1}$ Department of Pediatrics, Lincoln Medical \& Mental Health Center, Bronx, NY, United States

Corresponding Author: Aditya Badheka, 234 East 149th Street, 4-20 Bronx, NY, USA. 10451; Ph: 718-579-3026;

Fax: 718-579-4700; Email: dradityabadheka@gmail.com

Received: 29 October 2011

Accepted: 30 March 2012

Published: 01 February 2013 effects are of particular concern in children and adolescents due to the immediate and long term health risks associated with weight gain, including obesity, diabetes mellitus and hyperlipidemia.

Keywords: Quetiapine, Dyslipidemia, Hyperglycemia, Overweight, Pediatric

$* * * * * * * * *$

Carreño E, Dasari V, Badheka A, Yogananda S, DaveSharma S. Quetiapine associated dyslipidemia and hyperglycemia in a pediatric overweight patient. International Journal of Case Reports and Images 2013;4(2):120-122.

$* * * * * * * * *$

doi:10.5348/ijcri-2013-02-275-CR-9

\section{INTRODUCTION}

Medications such as a typical antipsychotics, like quetiapine can cause undesirable alterations in lipid profile and hyperglycemia. Adverse metabolic effects such as diabetes mellitus, lipid abnormalities and weight gain have increasingly been recognized with the use of the newer, so-called atypical antipsychotic drugs. There are several possible means by which an agent may induce hyperlipidemia. For most agents associated with lipid dysregulation these mechanisms are not clearly elucidated and that remains the case for antipsychoticinduced hyperlipidemia. Nonetheless, several biologically plausible hypotheses have been advanced focusing on weight gain, dietary changes and the development of glucose intolerance, to explain the high incidence of hyperlipidemia with certain antipsychotic medications $[1-5]$. One mechanism which is operative in some patients is the development of glucose intolerance, with a direct correlation found in a number 
of cases between the development of new onset Type-2 diabetes mellitus and elevated serum triglyceride (TG) levels, often with reversal of these problems upon discontinuation of the offending agent [6-8]. There is, however, ample evidence that the mechanism underlying hypertriglyceridemia related to atypical antipsychotic therapy is generally not dependent on glucose intolerance in light of the finding that increased serum TG or hypertriglyceridemia is prevalent in studies involving dibenzodiazepine-derived atypical antipsychotics. Hyperlipidemia is not mentioned in the side effects by manufacturers of quetiapine. The following is a case report of possible quetiapine-induced severe hyperlipidemia.

\section{CASE REPORT}

A 15-year-old overweight, Hispanic, asymptomatic male child was referred to the endocrine clinic by his primary care provider due to high serum cholesterol, triglyceride and glucose levels. No personal or family history of hypercholesterolemia, early cardiovascular event or diabetes was present. Past history was significant for autism, anxiety with depression and attention deficit disorder for which he was getting psychiatric care and was talking quetiapine $50 \mathrm{mg}$ daily for more than two years. Physical examination was significant for increased adiposity and BMI in the overweight range (85-95th percentile) with pubertal tanner stage 5. Levels at the time of presentation showed elevated fasting total cholesterol level of 388 $\mathrm{mg} / \mathrm{dL}$, triglyceride of $1420 \mathrm{mg} / \mathrm{dL}$ and HDL cholesterol of $46 \mathrm{mg} / \mathrm{dL}$, (LDL could not be calculated due to abnormally high TG).

Repeat levels of lipid panel and glucose showed similar results with elevated TGs and cholesterol two days later. LFTs showed elevated AST $47 \mathrm{IU} / \mathrm{dL}$ and ALT 105 IU/dL. Serum amylase, lipase, thyroid stimulating hormone, fasting insulin and urine microalbumin levels were normal. Electrocardiogram, echocardiogram and cardiac examination was normal. Quetiapine was discontinued immediately and replaced by aripiprazole by the psychiatrist. Lifestyle changes including dietary and exercise program was started. The laboratory tests repeated four months later showed normalizing serum cholesterol of $209 \mathrm{mg} / \mathrm{dL}$, triglycerides $261 \mathrm{mg} / \mathrm{dL}$, HDL $44 \mathrm{mg} / \mathrm{dL}$, LDL 113 $\mathrm{mg} / \mathrm{dL}$, AST $46 \mathrm{IU} / \mathrm{dL}$ and ALT $68 \mathrm{IU} / \mathrm{dL}$ with normal amylase, lipase, thyroid stimulating hormone and fasting insulin levels.

\section{DISCUSSION}

Atypical antipsychotics are increasingly prescribed to children and adolescents with neuropsychiatric disorders. Although their profile of potent antagonism at specific serotonin and dopamine receptors offers certain advantages compared with typical antipsychotics, their use has been associated with various adverse effects, including significant weight gain. No large controlled trials have been published quantifying the prevalence of adverse effects on glucoseinsulin homeostasis and lipid metabolism in patients receiving atypical antipsychotics [6]. Quetiapine binds to multiple receptors, including D1, D2, 5-HT2A, 5$\mathrm{HT} 1 \mathrm{~A}$, and $\mathrm{H} 1$ receptors. Less information is known about the properties of quetiapine in children and adolescents. Martin et al. published a 16 week openlabel trial of quetiapine (mean dosage $225 \mathrm{mg}$ /day range $10-350 \mathrm{mg} /$ day) in six patients (mean weight gain 2.9 $\mathrm{kg}$, range of wight gain $0.9-8.2 \mathrm{~kg}$ ). Mean weight gain was not statistically significant in the six patients as a whole [9]. Shaw et al. investigated the effectiveness and safety of quetiapine (mean dosage $47 \mathrm{mg} /$ day; range $300-800 \mathrm{mg} /$ day) in 15 children (mean age 15 years; range 13-17 years) with psychotic disorders. Mean weight gain over the 8 week open-label trial was $3.4 \mathrm{~kg}$ [6]. This adverse effect is of particular concern in children and adolescents, secondary to the immediate and long term health risks associated with weight gain, including obesity, diabetes mellitus, and hyperlipidemia $[11,7,8]$. We recommend that when evaluating the overall benefit-risk ratio of antipsychotics in children and adolescents, the practitioner needs to give careful consideration to possible metabolic disruptions or cardiovascular toxic effects, especially in individuals with comorbid metabolic conditions and those receiving concomitant psychotropic medications.

\section{CONCLUSION}

In summary, in past decades, the use of second generation and atypical anti-psychotics is increasing in young populations. Antipsychotics are associated with metabolic and cardiovascular-related adverse events in pediatric populations, especially when multiple antipsychotics or classes of psychotropic medications are co-prescribed. The cardio-metabolic adverse effects and tolerability is well known in adult populations but in pediatric population, cardio-metabolic adverse effects can be pronounced specially with long term use, obesity, use of multiple psychotropic medications, family history of coronary artery disease and diabetes. Pediatricians should closely follow such patients for their high risk of developing dyslipidemia and hyperglycemia. Routine laboratory studies including fasting lipid profile and glucose should be performed frequently.

\section{Acknowledgements}

Evelyn Erickson, MD

\section{Author Contributions}

Elizabeth Carreño - Substantial contributions to conception and design, Acquisition of data, Drafting the article, revising it critically for important intellectual content, Final approval of the version to be published 
Aditya Badheka - Substantial contributions to conception and design, Acquisition of data, Drafting the article, Revising it critically for important intellectual content, Final approval of the version to be published Vivekananda Dasari - Substantial contributions to conception and design, Acquisition of data, Drafting the article, Revising it critically for important intellectual content, Final approval of the version to be published Shanti Yogananda - Substantial contributions to conception and design, Acquisition of data, Drafting the article, revising it critically for important intellectual content, Final approval of the version to be published Swati Dave-Sharma - Substantial contributions to conception and design, Acquisition of data, Drafting the article, Revising it critically for important intellectual content, Final approval of the version to be published

\section{Guarantor}

The corresponding author is the guarantor of submission.

\section{Conflict of Interest}

Authors declare no conflict of interest.

\section{Copyright}

(C) Elizabeth Carreño et al. 2013; This article is distributed under the terms of Creative Commons Attribution 3.0 License which permits unrestricted use, distribution and reproduction in any means provided the original authors and original publisher are properly credited. (Please see www.ijcasereportsandimages.com /copyright-policy.php for more information.)

\section{REFERENCES}

1. Olfson M, Blanco C, Liu L, Moreno C, Laje G. National trends in the outpatient treatment of children and adolescents with antipsychotic drugs.
Arch Gen Psychiatry 2006;63(6):679-85.

2. McIntyre RS, Konarski JZ. Tolerability profiles of atypical antipsychotics in the treatment of bipolar disorder. J Clin Psychiatry 2005;66 Suppl 3:28-36.

3. McIntyre RS, Jerrell JM. Metabolic and Cardiovascular Adverse Events Associated With Antipsychotic Treatment in Children and Adolescents. Arch Pediatr Adolesc Med 2008;162(10):929-35.

4. Melkersson K, Dahl ML. Adverse Metabolic Effects Associated with Atypical Antipsychotics Literature Review and Clinical Implications. Drugs 2004;64(7):701-23.

5. Meyer JM. Novel antipsychotics and severe hyperlipidemia. J Clin Psychopharmacol 2001 Aug;21(4):369-74.

6. Domon SE, Webber JC. Hyperglycemia and hypertriglyceridemia secondary to olanzapine. J Child Adolesc Psychopharmacol 2001 Fall;11(3):285-8.

7. Ghaeli P, Dufresne RL. Elevated serum triglycerides with clozapine resolved with risperidone in four patients. Pharmacotherapy 1999 Sep;19(9): 1099-101.

8. Wetterling T. [Hyperlipidemia-side-effect of the treatment with an atypical antipsychotic (zotepine)?] Psychiatr Prax 2002 Nov;29(8):438-40.

9. Martin A, Koenig K, Scahill L, Bregman J. Open-label quetiapine in the treatment of children and adolescents with autistic disorder. J Child Adolesc Psychopharmacol 1999;9(2):99-107.

10. Shaw JA, Lewis JE, Pascal S, et al. A study of quetiapine: Efficacy and tolerability in psychotic adolescents. J Child Adolesc Psychopharmacol 2001;11(4):415-24.

11. Domon SE, Cargile CS. Quetiapine associated hyperglycemia and hypertriglyceridemia. J Am Acad Child Adolesc Psychiatry 2002 May;41(5):495-6.
Access full text article on other devices

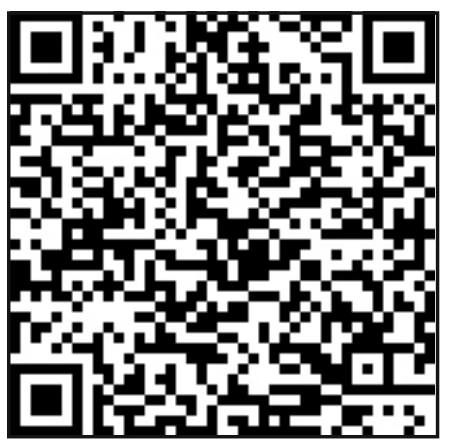

Access PDF of article on other devices

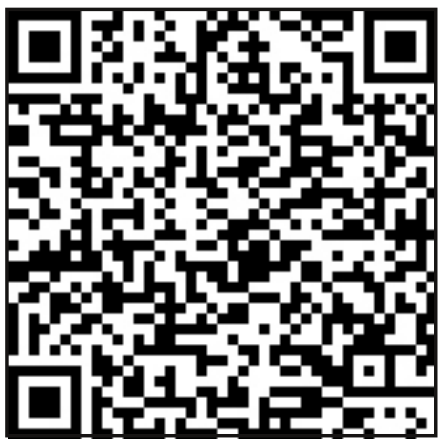

